\title{
A complex chromosome rearrangement with 10 breakpoints: tentative assignment of the locus for Williams syndrome to $4 \mathrm{q} 33 \rightarrow \mathrm{q} 35.1$
}

\author{
R Tupler, P Maraschio, A Gerardo, R Mainieri, G Lanzi, L Tiepolo
}

\begin{abstract}
An unbalanced complex chromosome rearrangement with 10 breakpoints resulting in four derivative chromosomes $(1,2$, 4 , and 11) was found in a girl with severe phenotypic abnormalities, many of which are characteristic of Williams syndrome. The patient was monosomic for the region 4 q33 $\rightarrow$ q35.1 and thus the mapping of the syndrome could tentatively be restricted to this region.
\end{abstract}

Complex chromosomal rearrangements (CCR) involve three or more breakpoints and may result in balanced or unbalanced karyotypes. An abnormal phenotype is always present in subjects with an unbalanced CCR and balanced ones are also often associated with phenotypic abnormalities.

Kousseff $e t$ al ${ }^{1}$ reviewed 19 CCR classified as group II (five or more breakpoints): among the 10 balanced cases only two had a normal phenotype and were familial while eight had various abnormalities with mental retardation. These abnormal phenotypes were probably the result of submicroscopical deletions or the involvement of one or more genes in a breakpoint. The highest number of breakpoints reported is eight.

We describe a female infant with a de novo unbalanced CCR with 10 breakpoints. She had severe mental retardation, congenital cardiac malformations, and facial features suggestive of Williams syndrome (WS). This syndrome
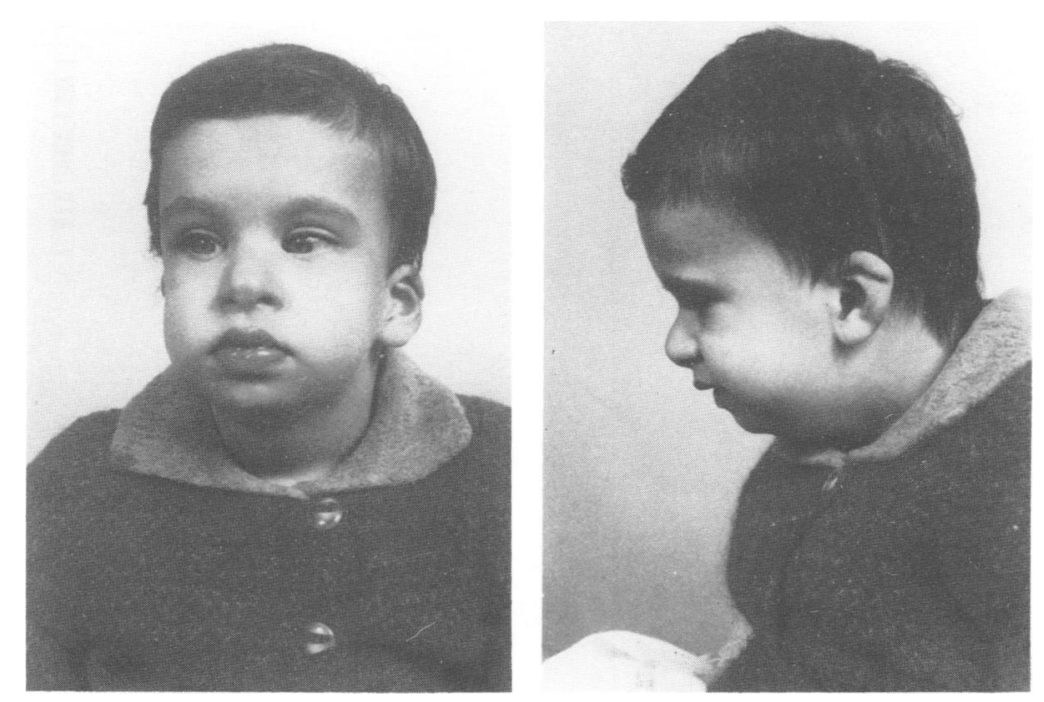

Figure 1 Patient at 3 years 5 months. was mapped to $4 \mathrm{q} 33 \rightarrow \mathrm{q} 35.1$ on the basis of a female infant showing clinical features of WS and monosomy of this region.

\section{Case report}

The proband was a female infant born at term on 25.11.87 after an uneventful pregnancy to a 22 year old primigravida. The father was 30 years old. Her weight was $2400 \mathrm{~g}(<3$ rd centile) and length was $50 \mathrm{~cm}$ (50th centile).

At birth severe cyanosis was present owing to tetralogy of Fallot. Before the initial cardiac surgery at five months she showed failure to thrive and slow feeding.

At 26 months she was admitted to the Child Neuropsychiatric Clinic because of severe psychomotor impairment. On clinical examination she showed weight, height, and head circumference below the 3 rd centile. She had a dolichocephalic cranium, malar flattening, fullness of the cheeks, depressed nasal bridge, prominent lower lip, and a large and open mouth, and a stellate iris pattern was observed (fig 1).

Psychomotor development was seriously impaired: she was unable to sit or stand unaided, or to roll from her back to her front. She showed diffuse muscular hypotrophy and hypotonia on neurological examination. Severe mental retardation was present with an autistic-like condition.

Heterotopic grey matter, enlarged fourth ventricle and cisterna magna, and pachygyria were detected on CT scan.

EEG showed aged related slow background activity, without abnormal excitability, correlated to the morphological abnormalities.

Phenotypic features of Williams syndrome present in our patient (based on the list of Preus ${ }^{2}$ ).

Low birth weight
Microcephaly
Dolichocephaly
Temporal depression
Molar flattening
Full cheeks
Swelling of periorbital region
Stellate iris
Depressed nasal bridge
Anteverted nares
Prominent philtrum
Large, open mouth
Prominent lower lip
High arched palate
Small teeth with malocclusion
Low set ears
Thick helix
Short and webbed neck
Low posterior hair line
Congenital cardiac anomaly (tetralogy of Fallot)
Short stature
Mental retardation




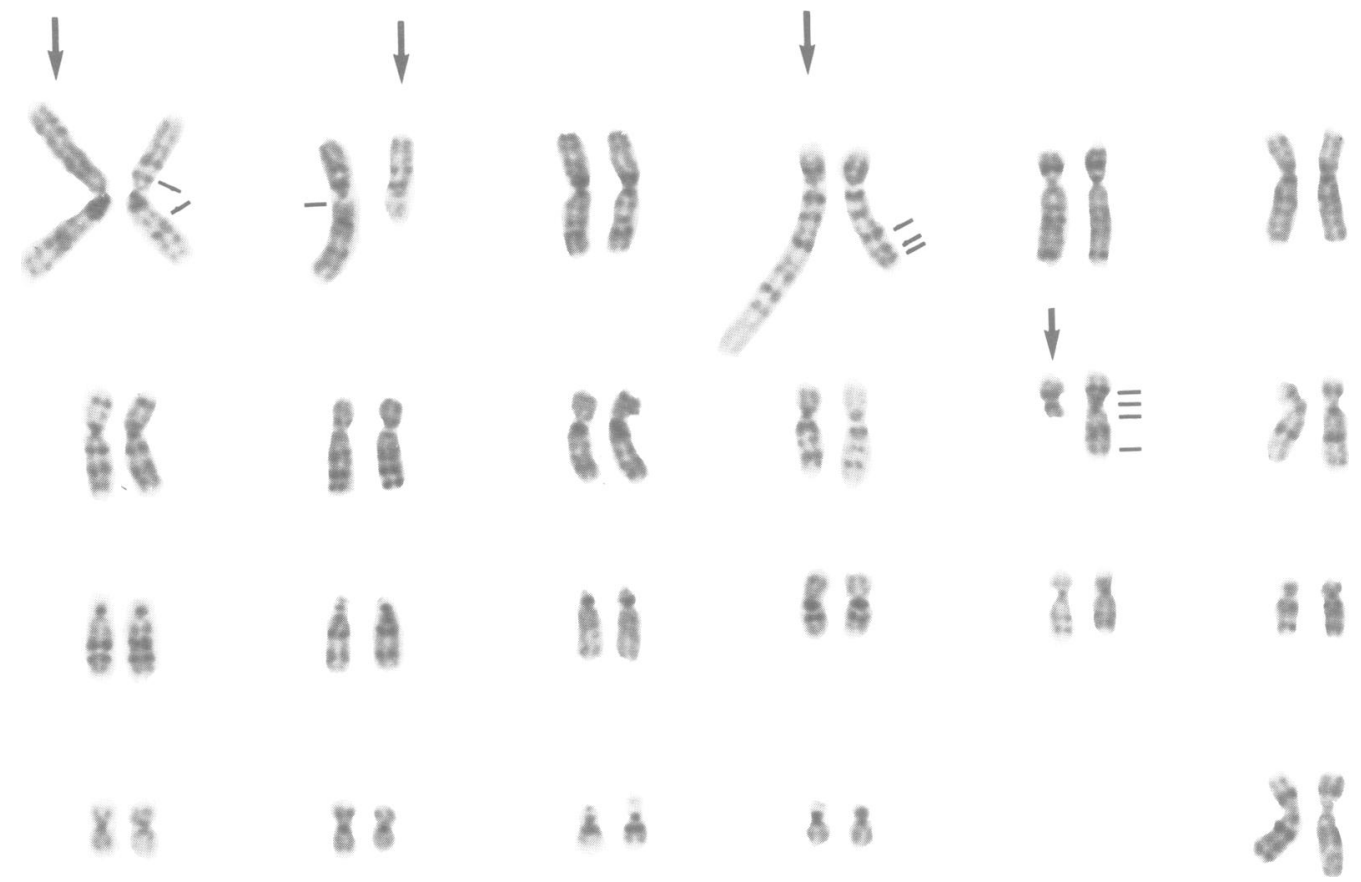

Figure 2 Karyotype of the patient. The arrows point to the rearranged chromosomes; the breakpoints are indicated by bars on the normal homologues.

The phenotypic features of WS present in the proband are listed in the table.

\section{CYTOGENETIC STUDIES}

Chromosome studies were performed on cultured lymphocytes and fibroblasts derived from a skin biopsy. GTG and high resolution banding ${ }^{3}$ showed a complex chromosomal rearrangement with 10 breakpoints involving chromosomes 1, 2, 4, and 11. According to our interpretation the rearrangement was balanced apart from a small region of the long arm of chromosome 4 which appeared to be missing. The karyotype, therefore, was $46, \mathrm{XX}, \mathrm{t}(1 ; 2 ; 4 ; 11)$ $(2 \mathrm{qter} \rightarrow 2 \mathrm{q} 11.2:: 1 \mathrm{p} 13.1 \rightarrow 1 \mathrm{q} 25:: 11 \mathrm{p} 13 \rightarrow 11 \mathrm{p}$ $11.11:: 1 \mathrm{q} 25 \rightarrow$ 1qter;2pter $\rightarrow$ 2q11.2::4q31.1 $\rightarrow$ 4q33::4q35.1 $\rightarrow$ 4qter;4pter $\rightarrow$ 4q31.1::11q13.1 $\rightarrow 11 \mathrm{q} 23:: 1 \mathrm{p} 13.1 \rightarrow 1 \mathrm{pter} ; 1 \mathrm{pter} \rightarrow 11 \mathrm{p} 13::$ $11 \mathrm{p} 11.11 \rightarrow 11 \mathrm{q} 13.1:: 11 \mathrm{q} 23 \rightarrow$ 11qter). The
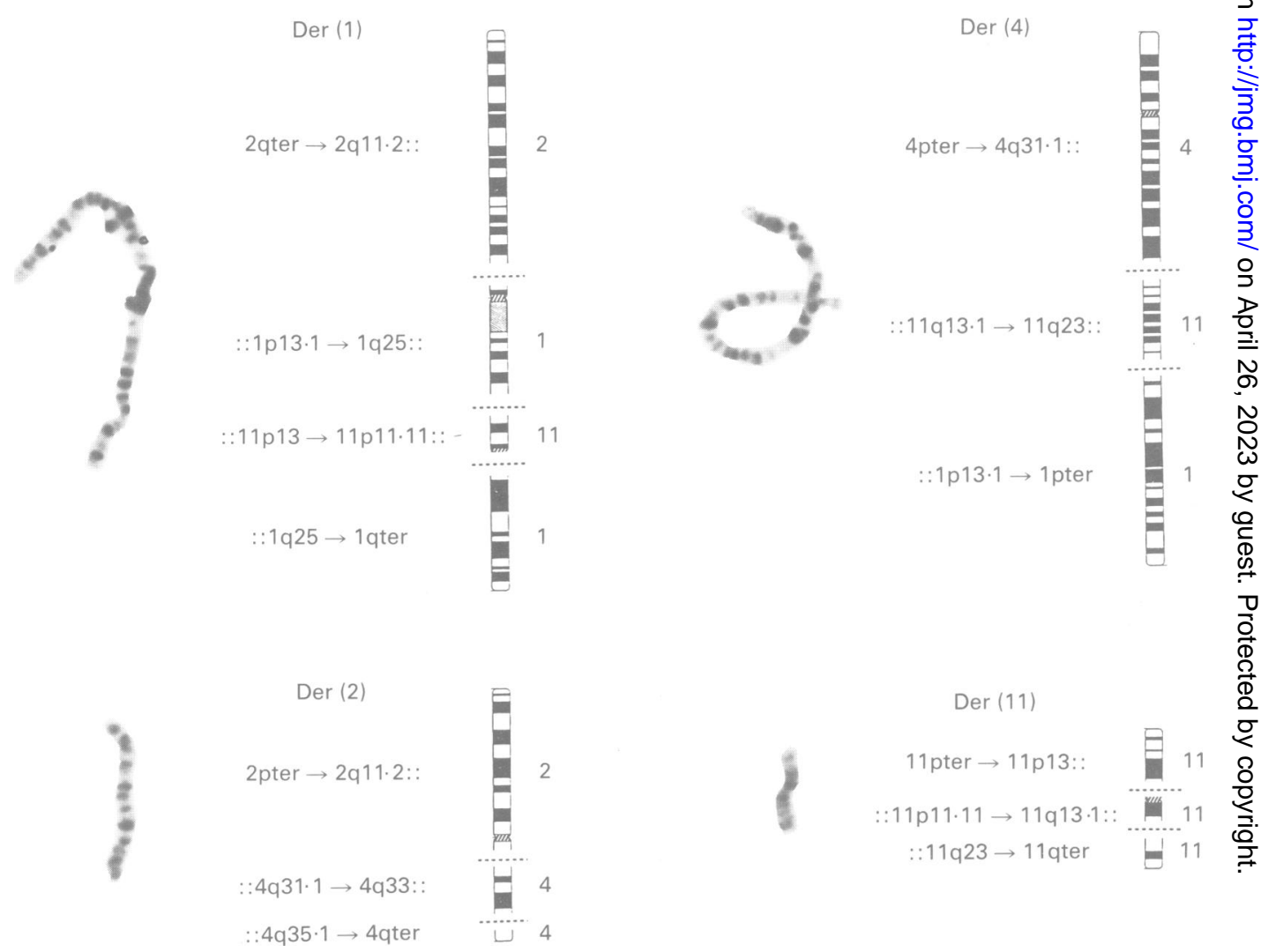

Figure 3 The four derivative chromosomes. 
Mother
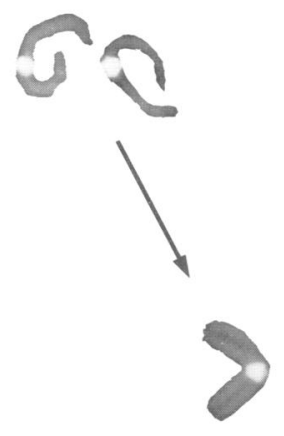

1

\section{Proband}

Figure 4 DA-DAPI staining of the chromosomes 1 of the parents and the proband. According to the size of the secondary constriction, the derivative chromosome 1 is of paternal origin.

karyotype was therefore unbalanced with monosomy for the $4 \mathrm{q} 33 \rightarrow \mathrm{q} 35.1$ region (figs 2 and 3).

The karyotypes of the parents were normal. The study of chromosomal polymorphisms with DA-DAPI banding showed the paternal origin of the abnormal chromosome 1 (fig 4).

Frozen fibroblasts from the proband are banked in Pavia, reference no 232/90.

\section{Discussion}

To trace back the series of events resulting in such a complex rearrangement is difficult. The only hint is the paternal origin of the chromosome 1 involved in the translocation, as indicated by the polymorphism of its secondary constriction after DA-DAPI staining. This chromosome was mutually exchanged with all the others involved in the rearrangement, and thus it is likely that the rearrangement took place in paternal gametogenesis.

Williams syndrome is an autosomal dominant disorder (McKusick 194050 ${ }^{4}$ ) characterised by typical dysmorphic facies with stellate iris, supravalvular aortic stenosis, and mental retardation. The clinical variability of the syndrome has led to the description of different nosological entities. Preus ${ }^{2}$ studied 52 possible cases of WS to define the natural history of the disease and to establish the clinical parameters necessary for diagnosis.

Our patient, in addition to most of the features listed in the table, shows other abnormalities consisting of severe psychomotor impairment and autistic-like behaviour correlated with the brain malformation, pachygyria and heterotopic grey matter, and tetralogy of Fallot. This clinical picture is probably the result of the complex chromosomal rearrangement with 10 breakpoints and monosomy $4 \mathrm{q} 33 \rightarrow \mathrm{q} 35.1$.

The patient described by Jefferson et $a l^{5}$ showed a terminal deletion of the long arm of chromosome 4 and the classical WS phenotype.

The presence in our patient of the characteristic signs of WS together with possible monosomy of $4 \mathrm{q} 33 \rightarrow \mathrm{q} 35.1$ suggests that the WS locus could map to this region.

However, in such a complex case we cannot rule out other possible additional chromosomal imbalances of small undetectable regions where the Williams syndrome gene could be located, or the localisation of this gene at one of the other breakpoints.

We are grateful to Professor M Fraccaro for critical reading of the manuscript.

1 Kousseff BG, Nichols P, Essig Y, Karen M, Weiss A Tedesco TA. Complex chromosome rearrangements an congenital anomalies. Am $\mathcal{f}$ Med Genet 1987;26:771-82.

2 Preus $M$. The Williams syndrome: objective definition and diagnosis. Clin Genet 1984;25:422-8.

3 Dutrillaux B, Viegas-Pequignot E. High resolution R and $G$ banding on the same preparation. Hum Genet 1981 57:93-5.

4 McKusick VA. Mendelian inheritance in man. 9th ed. Baltimore: The Johns Hopkins University Press, 1990.

5 Jefferson RD, Burn J, Gaunt KL, Hunter S, Davison EV. A terminal deletion of the long arm of chromosome 4 $46, X X, \operatorname{del}(4)(q 33)$, in an infant with phenotypic feature of Williams syndrome. $\mathcal{J}$ Med Genet 1986;23:474-80. 